\title{
Effect of Porphyromonas gingivalis infection on gut dysbiosis and resultant arthritis exacerbation in mouse model
}

Yuta Hamamoto', Kazuhisa Ouhara ${ }^{1 *}$ (D), Syuichi Munenaga ${ }^{1}$, Mikio Shoji², Tatsuhiko Ozawa ${ }^{3}$, Jyunzo Hisatsune ${ }^{4}$, Isamu Kado ${ }^{5}$, Mikihito Kajiya', Shinji Matsuda', Toshihisa Kawai ${ }^{6}$, Noriyoshi Mizuno', Tsuyoshi Fujita', Shintaro Hirata ${ }^{7}$, Kotaro Tanimoto ${ }^{5}$, Koji Nakayama ${ }^{2}$, Hiroyuki Kishi ${ }^{3}$, Eiji Sugiyama ${ }^{7}$ and Hidemi Kurihara ${ }^{1}$

\begin{abstract}
Background: Porphyromonas gingivalis $(\mathrm{Pg})$ infection causes periodontal disease and exacerbates rheumatoid arthritis (RA). It is reported that inoculation of periodontopathogenic bacteria (i.e., Pg) can alter gut microbiota composition in the animal models. Gut microbiota dysbiosis in human has shown strong associations with systemic diseases, including RA, diabetes mellitus, and inflammatory bowel disease. Therefore, this study investigated dysbiosis-mediated arthritis by Pg oral inoculation in an experimental arthritis model mouse.

Methods: Pg inoculation in the oral cavity twice a week for 6 weeks was performed to induce periodontitis in SKG mice. Concomitantly, a single intraperitoneal (i.p.) injection of laminarin (LA) was administered to induce experimental arthritis (Pg-LA mouse). Citrullinated protein (CP) and IL-6 levels in serum as well as periodontal, intestinal, and joint tissues were measured by ELISA. Gut microbiota composition was determined by pyrosequencing the $16 \mathrm{~s}$ ribosomal RNA genes after DNA purification of mouse feces. Fecal microbiota transplantation (FMT) was performed by transferring Pg-LA-derived feces to normal SKG mice. The effects of Pg peptidylarginine deiminase (PgPAD) on the level of citrullinated proteins and arthritis progression were determined using a PgPAD knockout mutant.

Results: Periodontal alveolar bone loss and IL-6 in gingival tissue were induced by Pg oral infection, as well as severe joint destruction, increased arthritis scores (AS), and both IL-6 and CP productions in serum, joint, and intestinal tissues. Distribution of Deferribacteres and S24-7 was decreased, while CP was significantly increased in gingiva, joint, and intestinal tissues of Pg-inoculated experimental arthritis mice compared to experimental arthritis mice without Pg inoculation. Further, FMT from Pg-inoculated experimental arthritis mice reproduced donor gut microbiota and resulted in severe joint destruction with increased IL-6 and CP production in joint and intestinal tissues. The average AS of FMT from Pg-inoculated experimental arthritis was much higher than that of donor mouse. However, inoculation of the PgPAD knockout mutant inhibited the elevation of arthritis scores and ACPA level in serum and reduced CP amount in gingival, joint, and intestinal tissues compared to Pg wild-type inoculation.
\end{abstract}

Conclusion: Pg oral infection affected gut microbiota dysbiosis and joint destruction via increased CP generation. Keywords: Porphyromonas gingivalis, Rheumatoid arthritis, Periodontitis, Citrullinated protein, Dysbiosis

\footnotetext{
* Correspondence: kouhara@hiroshima-u.ac.jp

'Department of Periodontal Medicine, Division of Applied Life Sciences,

Institute of Biomedical \& Health Sciences, Hiroshima University, 1-2-3 Kasumi, Minami-ku, Hiroshima 734-8553, Japan

Full list of author information is available at the end of the article
}

\section{$\triangle B M C$}

(c) The Author(s). 2020 Open Access This article is licensed under a Creative Commons Attribution 4.0 International License, which permits use, sharing, adaptation, distribution and reproduction in any medium or format, as long as you give appropriate credit to the original author(s) and the source, provide a link to the Creative Commons licence, and indicate if changes were made. The images or other third party material in this article are included in the article's Creative Commons licence, unless indicated otherwise in a credit line to the material. If material is not included in the article's Creative Commons licence and your intended use is not permitted by statutory regulation or exceeds the permitted use, you will need to obtain permission directly from the copyright holder. To view a copy of this licence, visit http://creativecommons.org/licenses/by/4.0/. The Creative Commons Public Domain Dedication waiver (http://creativecommons.org/publicdomain/zero/1.0/) applies to the data made available in this article, unless otherwise stated in a credit line to the data. 


\section{Background}

Periodontal disease (PD) is a highly prevalent infectious disease caused by periodontopathogenic bacteria, such as Porphyromonas gingivalis (Pg), Tannerella forsythia, and Treponema denticola, called red complex [1]. Host immune response against periodontopathogenic bacterial challenge causes severe destruction of dental supportive tissues, including cementum, periodontal ligament, and alveolar bone [2]. More specifically, local and systemic elevation of inflammatory cytokines, interleukin (IL)-6 and TNF- $\alpha$, affect PD progression through $\mathrm{T}$ cell activation [3]. Several studies have shown a strong correlation between PD, especially in the presence of Pg infection, and systemic disease, including non-alcoholic steatohepatitis (NASH), rheumatoid arthritis (RA), pre-birth, low weight birth, Alzheimer's disease, and Buerger disease [4-8].

RA is a systemic inflammatory autoimmune disease characterized by chronic inflammation and joint tissue destruction, potentially leading to functional disability [9]. The cause of RA is not fully understood, but a multifactorial pathogenesis, involving both environmental and genetic factors, has been widely accepted [10, 11]. Among the proposed environmental triggers, PD is currently considered as a risk factor for RA and may play a central role in disease initiation [12]. Furthermore, Pg, a Gram-negative anaerobic bacterium, has an important role in the pathogenic hypothesis linking RA and PD, owing to the production of peptidylarginine deiminase (PAD) and the strong protease gingipain [13]. PAD derived from $\mathrm{Pg}$ (PgPAD) can generate citrullinated proteins $(\mathrm{CP})$, in a manner similar to that of endogenous PAD (PADI4), which might result in the production of anti-citrullinated protein/peptide antibody (ACPA) [14].

We previously showed that systemic or oral Pg administration exacerbated RA-like experimental arthritis in a mouse model $[15,16]$. Specifically, with systemic Pg infection, bacterial proteins were detected in joint tissues showing arthritis outcomes [15]. However, these bacteriaderived proteins were not recovered from the affected arthritis joints in the Pg oral administration model [16], likely because the pathogenic effect of $\mathrm{Pg}$ in arthritis progression does not occur through direct bacterial stimulation. Therefore, there is a possibility that oral infection of Pg may impact on pathogenic outcomes of arthritis indirectly, possibly via altering gut microbiome which was recently found to have a remarkable effect on the manifestations of a number of systemic diseases [17, 18], including arthritis $[19,20]$. Indeed, Kato et al. reported that oral Pg administration can alter the gut microbiome [21].

Dysbiosis of gut microbiota was observed in RA patients, and alterations in the microbiome could distinguish RA patients from healthy individuals [22]. Clustering analysis at the genus level showed 4 different type of clusters. Each cluster was characterized by a high abundance of the following genera: Ruminococcus in cluster 1, Bacteroides in cluster 2, Blautia and Faecalibacterium in cluster 3 , and Prevotella in cluster 4. Cluster 4 , which was characterized by a high abundance of Prevotella, consisted of only the feces from RA patients. Changing in the amount and type of oral microbiota was also reported in RA patients [23]. Especially, an increased abundance of Prevotella, Aggregatibacter, Treponema, and Veillonella was reported. Furthermore, gut dysbiosis by oral inoculation of Pg was shown to contribute to the progression of experimental arthritis [22]. Periodontopathogenic bacteria, including $\mathrm{Pg}$, exists in dental plaque $\left(10^{10}\right.$ [colony-forming units (CFU)]/g) and the daily inoculation of $1-2 \mathrm{~L}$ saliva $\left(10^{9} \mathrm{CFU} / \mathrm{mL}\right)$ [24]. Therefore, it is plausible that swallowing mass of dental plaque and saliva with Pg can affect the composition of gut microbiota. To support this hypothesis, inoculated Pg can also survive in the acid conditions of gastric juice [25]. Previous studies showed that Pg could exacerbate the pathologic outcomes of experimental arthritis [15]. The mechanisms of Pg-induced arthritis included variant immune response in $\mathrm{T}$ cells, activation of osteoclastogenesis in joint tissues, systemic and local C5a elevation, and change in gut microbiota [16]. In humans, serum ACPA is used as a marker for RA diagnosis. Immune complex consisting of ACPA and CP is also an important molecule for joint destruction. Therefore, monitoring $\mathrm{CP}$ generation and ACPA synthesis may be good indicators of RA onset $[11,26]$. Of animal models used in RA studies, the SKG mouse was the only model for which reported elevated ACPA during progression of experimental arthritis was reported [27]. Taken together, these immune responses may be elicited after Pg inoculation and gut dysbiosis. Therefore, this study used the SKG model to investigate the effect of Pg inoculation on the progression of experimental arthritis, including an analysis of changes in gut microbiota and $\mathrm{CP}$ generation in serum and gingival, intestinal, and joint tissues.

\section{Methods \\ Preparation of bacteria}

Bacteria used in this study were purchased from ATCC. Pg W83 and 33277 were cultured on a sheep blood agar plate using the Anaeropack system (Mitsubishi Gas Chemical, Tokyo, Japan) at $37^{\circ} \mathrm{C}$. After a 2-day incubation, $\mathrm{Pg}$ was inoculated in $40 \mathrm{~mL}$ of trypticase soy broth supplemented with $1 \%$ yeast extract, hemin $(200 \mu \mathrm{g})$, and menadione $(20 \mu \mathrm{g})$. Bacteria were harvested in the exponential growth phase and washed with phosphate buffered saline (PBS) for experiments.

\section{Construction of Pg knockout mutant}

The Pg pad knockout mutant was constructed by double recombination of the target gene and $e r m F$ introduction, 
as previously described [28]. The targeting DNA was constructed as follows. The $0.5 \mathrm{~kb}$-upstream and $0.5 \mathrm{~kb}$ downstream regions of the pad gene were amplified with two pairs of primers (pad-Up-F: [GGTCTCACAC GAGAGGATACTATGGTCTAT]/pad-Up-R: [CGGGGGATCCTGTTTGATATGTTTTATGAT]; pad-Dw-F: [TAGGGGATCCGGGGCCTTATTTGAGA ATAC]/pad-Dw-R: [AGCAGAGGTTACGAGCTTAACCAGAGATGC], where 'Up,' 'Dw,' ' $F$,' and ' $R$ ' indicate upstream, downstream, forward, and reverse, respectively) using the genome of ATCC 33277 as a template. The ermF region in the ermF DNA cassette was amplified with ermF-F: [ATATCAAACAGGATCCCCCGATAGCTTCCG]/ermF-R: [ATAAGGCCCCGGATCCCC TACGAAGGATGA] using the genome of $g t f F$ (PGN 1668)::ermF mutant (KDP611) as a template [29]. Using the three purified products, PCR was performed with pad-Up-F/ pad-Dw-R. Finally, the desired PCR product was purified and introduced into Pg ATCC 33277 by electroporation. Transformants were selected on blood agar plates containing $10 \mu \mathrm{g} / \mathrm{mL}$ erythromycin. The mutation of target gene was confirmed by PCR using padUp-R/pad-Dw-R. Correct ermF gene insertion of Pg pad knockout mutant was also verified by sequencing.

\section{Generation of Pg-infected experimental arthritis mouse model}

All animal experimental procedures employed in this study were approved by the Ethics Committee of Hiroshima University (approval No. A16-33) and performed as previously reported [16]. Briefly, SKG mice were maintained under specific pathogen free (SPF) condition until inoculation of bacteria and feces. The food used in this study was a regular chow for mouse with gamma irradiation (Type MF; Oriental Yeast Co., Ltd., Tokyo, Japan). Laminarin derived from Laminaria digitata (LA) was purchased from Sigma-Aldrich (L9634, St. Louis, MO, USA). LA was dissolved in PBS at $100 \mathrm{mg} / \mathrm{mL}$. To induce experimental arthritis, LA (10 mg/100 $\mu \mathrm{L} /$ mouse) was administered to SKG mice by an intraperitoneal (i.p.) injection. $\mathrm{Pg}$ was also inoculated $\left(10^{8}\right.$ bacterial cells $/ 50 \mu \mathrm{L}$ in $2 \%$ carboxymethylcellulose (CMC) solution) twice a week for 6 weeks. LA injection and Pg inoculation were started the same day. The same volume of CMC solution was inoculated as a negative control. Mice were divided into 3-4 groups (5 or 6 mice per group) in each experiment (Ctrl: CMC inoculation, LA: LA i.p injection, Pg: Pg oral inoculation, Pg/LA: LA i.p. injection with Pg oral inoculation, LA-FMT: LA i.p. injection with FMT from the LA group, Pg/LA-FMT: LA i.p. injection with FMT from the Pg/LA group). Power calculation was performed by the same method as that reported in our previous publication that used the SKG mouse model to evaluate the effects of $\mathrm{Pg}$ on the induction of arthritis [16]. In the above noted publication, arthritic score of Pg+LA treated group and that of LA alone group showed $3.6+1.4$ and $1.0+0.3$, respectively (average $+\mathrm{SD}$ ). Accordingly, the sample size required for the in vivo experiments, i.e., $n=5$ /group, was determined based on $80 \%$ power at 0.05 significance.

\section{Evaluation of alveolar bone level in mouse}

The alveolar bone level (ABL) of SKG mice was evaluated by Kawai's methods described previously [30]. Briefly, after methylene blue staining (Sigma-Aldrich) for $10 \mathrm{~min}$, the upper molar jaw was washed with PBS three times. The length of the blue-stained root surface of all molar teeth from the enamel-cement junction to the top of the alveolar bone was measured. Differences between treated mice and the control group (no treatment) were evaluated.

\section{Clinical assessment of arthritis score (AS) in SKG mice}

Joint swelling was monitored by inspection and scored as follows: 0 , no joint swelling; 0.1, swelling of one finger joint; 0.5 , mild swelling of the wrist or ankle; 1.0, severe swelling of the wrist or ankle. Scores for all digits, wrists, and ankles were totaled for each mouse, as reported previously [31].

\section{IL-6 measurement in mouse tissue}

Serum and tissues from gingiva, leg joint, small intestine, and large intestine were collected from each mouse and homogenized by cool-mill (\#TK-CM20S, Tokken, Inc., Chiba, Japan) in RIPA Lysis and Extraction Buffer (\#89900, Thermo Fisher Scientific, Tokyo, Japan, $100 \mathrm{mg}$ tissue $/ 100 \mu \mathrm{L}$ ) with $0.1 \%$ phenylmethanesulfonyl fluoride (PMSF, Sigma-Aldrich) and $1 \%$ proteinase inhibitor cocktail (\#87786, Thermo Fisher Scientific). The sera were diluted four times by PBS. Supernatants of the tissue homogenates and sera were used for IL-6 measurement by ELISA (for mouse IL-6, \#431304; BioLegend Inc., San Diego, CA, USA), according to the manufacturer's instructions. Briefly, a solid-phase anti-IL-6 monoclonal antibody (diluted in coating buffer to a final concentration of $1 \mu \mathrm{g} / \mathrm{mL}$ ) was coated onto a 96-well ELISA plate (BD Falcon, Franklin Lakes, NJ, USA) for target capture. After blocking each well with $1 \%$ BSA in PBS supplemented with $0.05 \%$ Tween 20 (PBST), the sample or standard (diluted in PBST from $1 \mathrm{ng} / \mathrm{mL}$ to zero) was applied to each well. After application of the detection antibody (diluted in PBST to a final concentration of $1 \mu \mathrm{g} / \mathrm{mL}$ ), horseradish peroxidase (HRP) conjugated with anti-IgG (2000-fold dilution in PBST) was applied to the wells. Colorimetric reactions were developed with o-phenylenediamine (Sigma-Aldrich) in the presence of $0.02 \% \mathrm{H}_{2} \mathrm{O}_{2}$. Color development was stopped with $\mathrm{H}_{2} \mathrm{SO}_{4}(2 \mathrm{~N})$ and measured using an ELISA 
reader $\left(\mathrm{OD}_{405}\right.$, Varioskan LUX). The actual concentration of the target was calibrated by referring to a standard curve prepared by serial dilutions. Each sample was examined in triplicate wells of a 96-well ELISA plate. The limits of mouse IL- 6 detection for each analyte were $15.6 \mathrm{pg} / \mathrm{mL}$.

\section{Detection of citrullinated protein in mouse tissue}

Serum and homogenized tissues from the gingiva, leg joint, small intestine, and large intestine were diluted in sodium bicarbonate buffer $(\mathrm{pH} \mathrm{9.4,} 10 \mu \mathrm{g} / \mathrm{mL})$ and coated onto a 96-well ELISA plate. After blocking each well with $1 \%$ BSA and sucrose in PBST, anti-citrulline monoclonal IgG (clone CCP-Ab1: generated from B cells isolated from an RA patient, $10 \mu \mathrm{g} / \mathrm{mL}$ ) was reacted in PBST at room temperature for $2 \mathrm{~h}$ [32]. Subsequently, anti-human IgG conjugated with HRP (2000-fold dilution in PBST) was applied to the wells. Colorimetric reactions were performed by the same method as that was used for IL- 6 measurement.

\section{Measurement of serum ACPA level}

Sera from each group were collected to measure ACPA levels. Briefly, cyclic citrullinated peptides (CCP) (Orgentec, Chicago, IL, USA, diluted in PBS pH 7.2 at final concentration of $0.5 \mu \mathrm{g} / \mathrm{mL}$ ) were pre-coated onto a 96-well ELISA plate (BD Falcon, Franklin Lakes, NJ, USA) for target capture. After blocking each well with 1\% BSA in PBST, serum (4-fold dilution) or standard (diluted in PBST from $1 \mathrm{ng} / \mathrm{mL}$ to zero) was applied to each well. After application of the detection antibody (diluted in PBST to a final concentration of $1 \mu \mathrm{g} / \mathrm{mL}$ ), anti-IgG conjugated with HRP (2000-fold dilution in PBST) was applied to the wells. Colorimetric reactions were developed using the same protocol as that was used for IL-6 detection. The actual concentration of the target was calibrated by referring to a standard curve prepared by serial dilutions. Each sample was examined in triplicate wells of a 96-well ELISA plate.

\section{Histological observation}

Ankle joints were fixed in $4 \%$ buffered formalin and embedded in paraffin wax. Subsequently, the tissues were sliced at a thickness of $7 \mu \mathrm{m}$ and mounted on glass slides. The paraffin-embedded sections were stained with hematoxylin and eosin (H\&E). The severity of inflammation and cartilage damage was scored in reference to the published criteria [33].

\section{Statistical analysis}

All experiments were performed at least three times independently. Data are expressed as mean \pm standard deviation (SD). Statistical analyses between two groups were performed using Mann-Whitney $U$ test for non- normal distribution. For multiple comparisons, the Tukey-Kramer test or Bonferroni-corrected MannWhitney $U$ test was used. $P<0.05$ was considered significant.

\section{Metagenomics of gut microbiota}

Bacterial DNA was extracted from feces as described previously [34]. In brief, feces were collected from all groups of mice (Ctrl, LA, Pg, Pg/LA, LA-FMT, Pg/LAFMT) before treatment as a control (day 0 ) and at the end of experiments (day 42). The bacterial composition of day 0 was confirmed the no significant difference. The collected feces were suspended in PBS (10\% sodium dodecyl sulfate, $10 \mathrm{mM}$ Tris- $\mathrm{HCl}$, and $1 \mathrm{mM}$ EDTA, $\mathrm{pH}$ 8.0), and the bacterial DNA was purified using the DNeasy PowerSoil Kit according to the manufacturer's instructions (with range of total DNA from $349.8 \mathrm{ng} / \mu \mathrm{l}$ to $611.8 \mathrm{ng} / \mu \mathrm{l}, \# 12888-100$ QIAGEN, Germantown, MD, USA). The concentration and quality of purified DNA was determined by Qubit ${ }^{\circ}$ Fluorometer (Thermo Fisher Scientific, Tokyo, Japan) and gel electrophoresis. As the positive control of bacterial DNA, Pg W83 was also purified with the same protocol. In order to detect Pg DNA from the tissues of SKG mouse, DNA was purified from each tissue by GenCheck DNA purification kit (FASMAC Co., Ltd., Hokkaido, Japan). Then, 16s rRNA gene was detected by PCR as shown previously [35].

Afterwards, the V4 variable region (515F-806R) of bacterial $16 \mathrm{~S}$ rRNA genes was amplified and used for sequencing on an Illumina Miseq as previously described [36]. Each reaction mixture contained $15 \mathrm{pmol}$ of each primer (16S Amplicon PCR Forward Primer 5'-TCGTCGGCAG CGTCAGATGTGTATAAGAGACAGCCTACGGGNGG CWGCAG, 16S Amplicon PCR Reverse Primer 5'-GTCT CGTGGGCTCGGAGATGTGTATAAGAGACAGGACT ACHVGGGTATCTAATCC), KAPA HiFi HotStart ReadyMix $(\times 2)$ (Hokkaido System Science Co., Ltd., Sapporo, Japan), $50 \mathrm{ng}$ extracted DNA, and sterilized water to reach a final volume of $50 \mu \mathrm{L}$. PCR conditions were as follows: $95^{\circ} \mathrm{C}$ for $3 \mathrm{~min} ; 25$ cycles of $95^{\circ} \mathrm{C}$ for $30 \mathrm{~s}, 55^{\circ} \mathrm{C}$ for $30 \mathrm{~s}$, and $72^{\circ} \mathrm{C}$ for $30 \mathrm{~min}$; and completed by the final extension at $72^{\circ} \mathrm{C}$ for $5 \mathrm{~min}$. The PCR product was purified by AMPure XP (Beckman Coulter, Inc., Brea, CA, USA) and quantified using a Quant-iT PicoGreen ds DNA Assay Kit (Life Technologies Japan Ltd., Tokyo, Japan). Mixed samples were prepared by pooling approximately equal amounts of PCR amplicons from each sample. The pooled library was analyzed with an Agilent High Sensitivity DNA Kit on an Agilent 2100 Bioanalyzer (Agilent Technologies, Santa Clara, CA). Real-time PCR was performed on the pooled library using a KAPA Library Quantification Kit for Illumina, following the manufacturer's protocols, and a sample library with a $20 \%$ denatured PhiX spike-in was sequenced by Miseq using a 600 cycles kit to 
obtain $2 \times 250 \mathrm{bp}$ paired-end reads. Taxonomic assignments and estimation of relative abundance of sequencing data were performed using the analysis pipeline of the QIIME software package [37]. An operational taxonomic unit (OTU) was defined at $97 \%$ similarity. OTUs indicating relative abundance under $0.05 \%$ were filtered to eliminate noise. The OTU was assigned a taxonomy based on a comparison with the Silva database using UCLUST [38].

\section{Fecal microbiota transmission (FMT)}

Fresh mouse feces from the LA and Pg/LA group were collected 6 weeks after when Pg inoculation was started. Collected feces from the same group (two fecal pellets [10-30 mg/fecal pellet] from each mouse; $n=6$ /group) were pooled and homogenized in anaerobic resuspension buffer $(10 \mathrm{mg} / \mathrm{ml}, 2 \%$ Lab-Lemco powder, $\quad 0.1 \%$ L-cysteine, $\quad 0.045 \% \quad \mathrm{KH}_{2} \mathrm{PO}_{4}, \quad 0.09 \%$ $\mathrm{NaCl}, \quad 0.045 \% \quad\left(\mathrm{NH}_{4}\right)_{2} \mathrm{SO}_{4}, \quad 0.0045 \% \quad \mathrm{CaCl}_{2}, \quad 0.0045 \%$ $\mathrm{MgSO}_{4}$ and $40 \%$ glycerol in $1000 \mathrm{~mL}$ ) and kept at $80^{\circ} \mathrm{C}$ until used. Before FMT, SKG mice were treated with antibiotics (ampicillin (Sigma-Aldrich) $1 \mathrm{~g} / \mathrm{L}$, metronidazole (Sigma-Aldrich) $1 \mathrm{~g} / \mathrm{L}$, neomycin (Sigma-Aldrich) $1 \mathrm{~g} / \mathrm{L}$, vancomycin (Sigma-Aldrich) $0.5 \mathrm{~g} / \mathrm{L}$ ) dissolved in water for 1 week. At this point, feces were collected prior to FMT and the changing of gut microbial composition was confirmed. Female SKG mice (6-8 weeks old) received gastric intubation of $250 \mu \mathrm{L}$ of the fecal suspensions from donor mice twice in a week. Subsequently, these mice were intraperitoneally injected with LA $(100 \mu \mathrm{g})$ at 1 week after bacterial gastric intubation. AS and ankle thickness were monitored every week for 6 weeks after injection. Six weeks after FMT, feces of two recipient groups that received LA and $\mathrm{Pg} / \mathrm{LA}(n=5$ /group) along with respective FMT were collected independently and microbiota composition of the each mouse was analyzed by next generation DNA sequencing of bacterial $16 \mathrm{~s}$ rRNA.

\section{Western blotting}

Homogenized tissues from the leg joint and gingival tissue were applied to a $12 \%$ SDS-polyacrylamide gel for electrophoresis and electronically transferred onto nitrocellulose membranes (BioRad Laboratories, CA, USA). The membrane was blocked with $1 \%$ non-fat dried milk at room temperature for $1 \mathrm{~h}$ and then reacted with antiPADI2 rabbit monoclonal IgG $(10 \mu \mathrm{g} / \mathrm{ml}, 12,110-1$-AP, PROTEINTECH JAPAN, Japan), anti-PADI4 rabbit monoclonal IgG (10 $\mu \mathrm{g} / \mathrm{ml}$, EPR20706, abcam, Japan), or anti-Pg rat serum (1000 folds dilution) in PBST at $4{ }^{\circ} \mathrm{C}$ for $12 \mathrm{~h}$. The membrane was incubated with HRPconjugated with sheep anti-rabbit IgG or sheep anti-rat IgG in PBST at room temperature for $1 \mathrm{~h}$. Immunodetection was performed according to the manual supplied with ECL Plus Western blotting reagents (GE Healthcare Life Sciences, Japan). As a control, the amount of Glyceraldehyde 3-phosphate dehydrogenase (GAPDH) was detected by anti-GAPDH antibody $(10 \mu \mathrm{g} / \mathrm{ml}$, HRP60004, PROTEINTECH JAPAN, Japan) The density of target PADI2 and PADI4 bands was measured by $\mathrm{NIH}$ Image-J software.

\section{Results}

Evaluation of periodontal tissue in experimental arthritis model mice with Pg infection

Oral infection of Pg induced alveolar bone loss in the Pg and Pg/LA groups (Fig. S1a and b). However, the Ctrl and LA groups did not show any alveolar bone loss. Additionally, IL-6 production in mouse gingival tissue was measured and it was found to be increased in the Pg and $\mathrm{Pg} / \mathrm{LA}$ groups compared to the $\mathrm{Ctrl}$ group (Ctrl $92.2 \mathrm{pg} / 10 \mathrm{mg}$ tissue, Pg $318.3 \mathrm{pg} / 10 \mathrm{mg}$ tissue, Pg/LA $319.4 \mathrm{pg} / 10 \mathrm{mg}$ tissue). Although it is well established that RA affects periodontitis clinically, and that especially the treatment of RA resulted in the improvement of periodontitis [39], IL-6 production in mouse gingival tissue of the LA group was similar to that of the Ctrl group (Fig. 1a). In the purified DNA from serum, feces, gingival tissue, tongue, lung, stomach, small intestine, and large intestine of the Pg group, Pg-derived DNA was only detected from gingival tissue, tongue, and lung tissue (Fig. S1d).

\section{Assessment of experimental arthritis in mice}

To assess experimental arthritis in mice with Pg oral inoculation, serum and joint tissues were examined. AS increased in the LA group 5 weeks after LA injection. Four weeks after LA injection and Pg inoculation, AS score of the Pg/LA group was also significantly elevated matching the results from our previous report [16]. However, AS of Ctrl and Pg groups did not show the induction of joint swelling (Fig. S1c). H\&E-stained sections of joint tissues in the LA group showed mild infiltration of immune cells and growth of granulation tissue. Furthermore, severe inflammation and pannus formation in the $\mathrm{Pg} / \mathrm{LA}$ group were observed (Fig. 1b-e). Inflammation and cartilage damage in joint tissue were more severe in the $\mathrm{Pg} / \mathrm{LA}$ group compared to other groups (inflammation score $2.75 \pm 0.43$, cartilage damage score $2.50 \pm$ 0.50) (Fig. 1f, g). The serum ACPA levels and IL-6 levels in serum and joint tissues were elevated in the LA, Pg, and $\mathrm{Pg} / \mathrm{LA}$ groups compared to the Ctrl group, with the highest levels in the Pg/LA group (Fig. $1 \mathrm{~h}-\mathrm{j}$ ). The most elevated level of serum IL-6 in the Pg/LA group among four groups corresponded to our former report that examined the same panel of experiment groups using SKG mouse model [16]. In the present study, although serum IL-6 in the group of LA alone was lower than that of Pg/ 


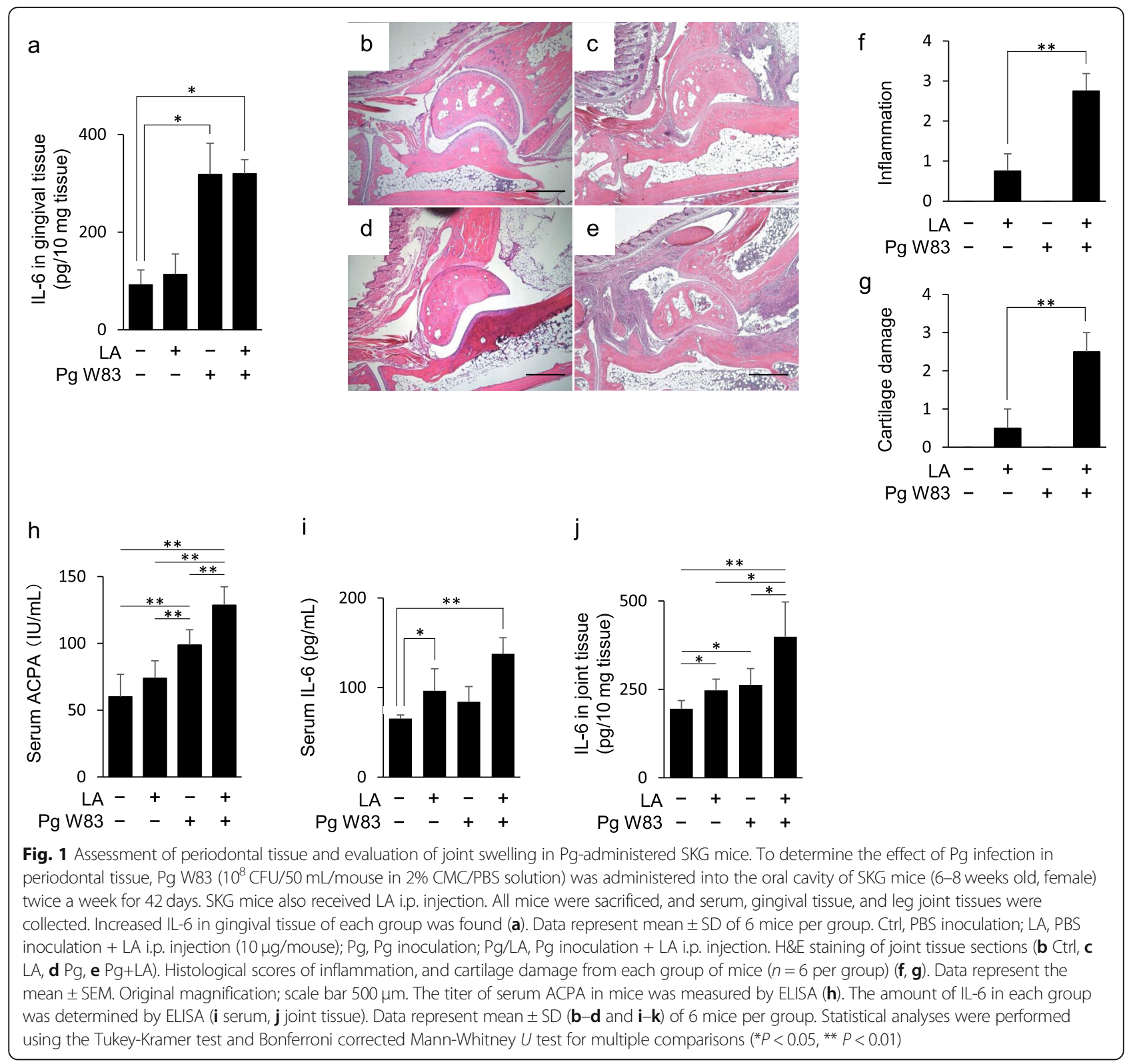

LA group, there was no significant difference between those two group, contrast to the previous study that detected significant difference in serum IL-6 between corresponding two groups [16] Interestingly, the baseline level of serum IL-6 was also higher than previous study [16]. As the result of the ZAP-70 gene mutation in T cells, arthritis occurs spontaneously without LA [40], while the cellular source of IL-6 in SKG appears to be macrophages [41] which can be affected by the PAMPs released from oral and gut microbiome of the mice. In this present study, the SKG mice were bread in the vivarium of Hiroshima University contrast to the former study that used SKG mice bred in Japan Clea corp [16]., although some discrepancy in the productions of serum
IL-6 between present and former of our study using SKG RA mouse model may be attributed to the distinct microbiomes established in the mice at two different facilities.

\section{Effect of $\mathrm{Pg}$ oral administration on gut microbiota composition}

The composition and abundance of gut microbiota in mouse fecal samples were assessed by next-generation sequencing of $16 \mathrm{~s}$ rRNA genes. The relative abundance in phylum level in the LA, Pg, and Pg/LA groups was changed compared to the Ctrl group (Fig. 2a). Further, the LA, Pg, and Pg/LA groups showed increased relative abundance at the order level of Bacteroides compared to 


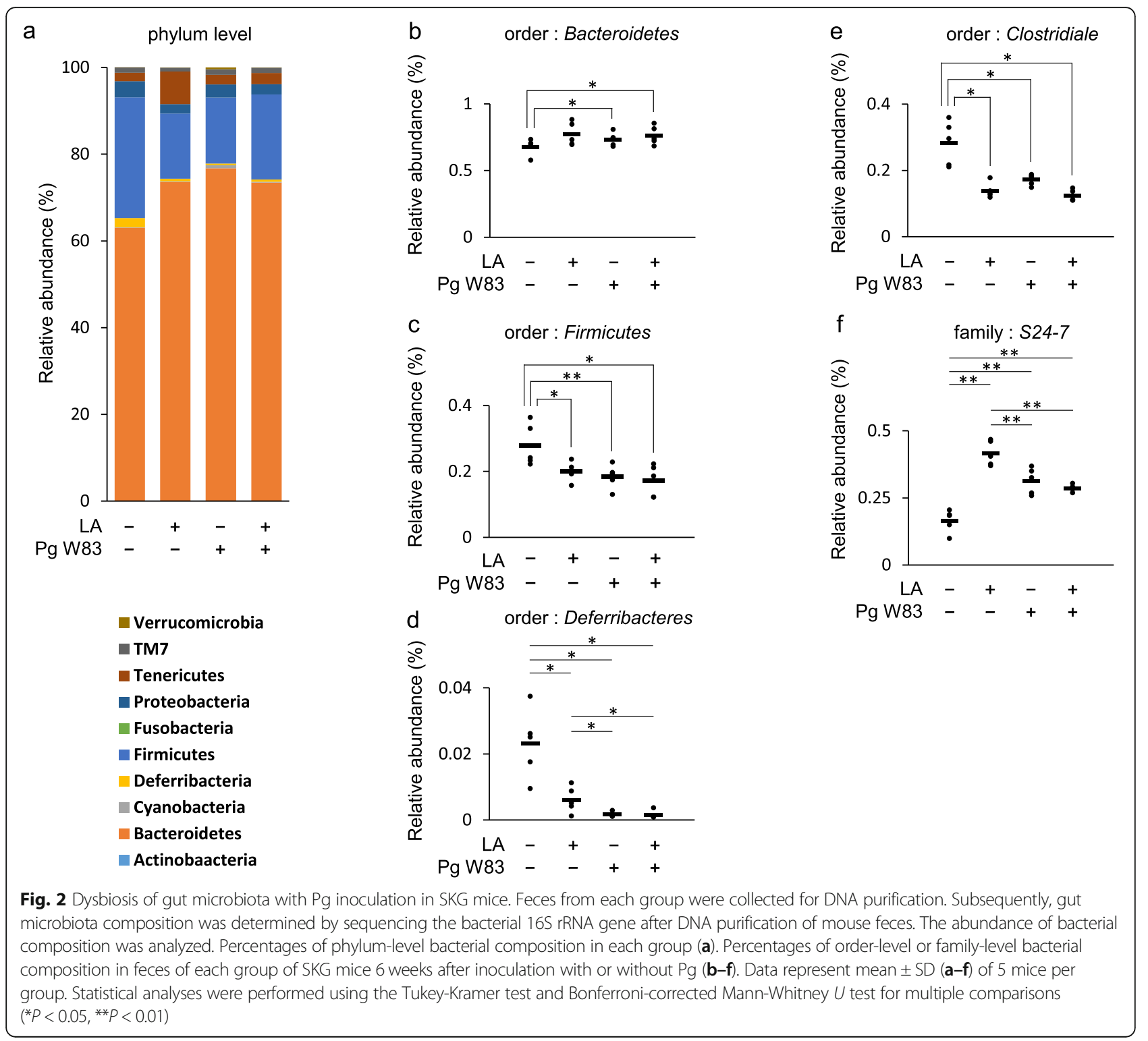

controls (Fig. 2b). Conversely, the relative abundance of Firmicutes, Deferribacteres, and Clostridiales was decreased in LA, Pg, and Pg/LA groups compared to Ctrl (Fig. 2c-e). The relative abundance of Deferribacteres in the Pg and Pg/LA groups decreased compared to the LA group (Fig. 2d). At the family level, the relative abundance of S24-7 in the LA, Pg, and Pg/LA groups was significantly increased compared to Ctrl, but the relative abundance of S24-7 in the Pg and Pg/LA groups was lower than that in LA (Fig. 2f).

\section{Effect of Pg oral administration on gut inflammation}

To assess gut inflammation, IL-6 production in the tissues of the small and large intestine was measured (Fig. 3a, b). IL-6 production in the small intestine of the $\mathrm{Pg} / \mathrm{LA}$ group was remarkably elevated compared to other groups (Pg/LA group, $184.8 \mathrm{pg} / 50 \mathrm{mg}$ tissue). In the Pg group, IL-6 production in the small intestine was slightly increased compared to the Ctrl group (Ctrl group, $36.4 \mathrm{pg} / 50 \mathrm{mg}$ tissue; Pg group, $67.8 \mathrm{pg} / 50 \mathrm{mg}$ tissue). Further, IL-6 production in the large intestine of the $\mathrm{Pg} / \mathrm{LA}$ and $\mathrm{Pg}$ groups was remarkably elevated compared to other groups (Pg/LA group, $521.9 \mathrm{pg} / 50$ $\mathrm{mg}$ tissue; Pg group, $370.7 \mathrm{pg} / 50 \mathrm{mg}$ tissue). IL-6 production in the small and large intestine of the LA group was not elevated after 6 weeks. This could be attributed to the induction of experimental arthritis by the single injection of LA takes at least 20 weeks [27]. Thus, at 6 week, it is too early to see the effects of proinflammatory factors produced in arthritis lesion on the level of IL- 6 in other tissue, especially in gut, via systemic dissemination. 


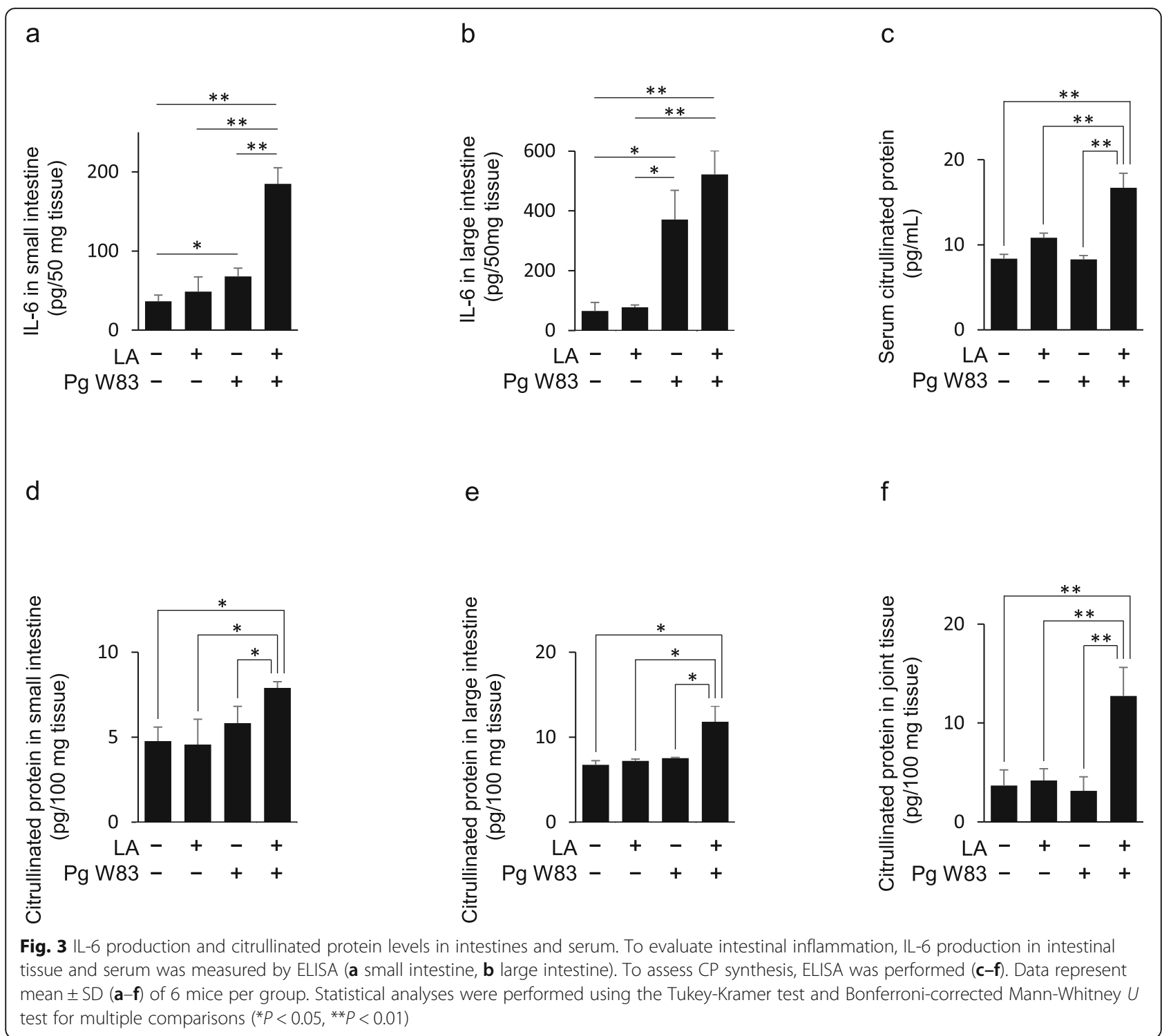

Effect of Pg oral administration on CP generation

ACPA-activated inflammation depends on $\mathrm{CP}$ generation [11]. Therefore, CP generation in serum and tissues of small and large intestines, and joint was measured by ELISA (Fig. 3c-f). Elevated CP in the Pg/ LA group was observed in small intestinal tissue, large intestinal tissue, serum, and joint tissue (1.73-, 1.64-, 3.03-, and 1.65-fold compared to the LA group). CP generation in the small and large intestine of the LA group was not elevated after 6 weeks for the same reason that IL-6 was not elevated in gut tissue, as noted above same reason as the IL-6 elevation in gut tissue.

\section{Effect of FMT on onset of experimental arthritis}

Pg oral inoculation and LA injection in SKG mice induced RA-like experimental arthritis. Furthermore, Pg inoculation resulted in changes in gut microbiota and inflammation of gut tissue in this model. To determine if gut microbiota change induced by Pg inoculation were important in the exacerbation of experimental arthritis, FMT of feces derived from LA and Pg/LA donor mice was performed on LA-injected recipient mice. Feces were collected from the donor mice (LA and Pg/LA mice: 6 weeks after LA injection with or without Pg inoculation). The feces from recipient mice were collected 6 weeks after the LA injection with FMT. The composition and abundance of gut microbiota in mouse fecal samples from donor mice and recipient mice were assessed by percentages of order-level and phylum-level bacterial composition (Fig. 4a-d). Composition at the phylum level of LA-FMT and $\mathrm{Pg} / \mathrm{LA}-\mathrm{FMT}$ recipient groups was similar to that of the LA and Pg/LA donor groups, respectively. Although the relative abundance of Bacteroides or Firmicutes showed the difference between sham group and Pg oral 


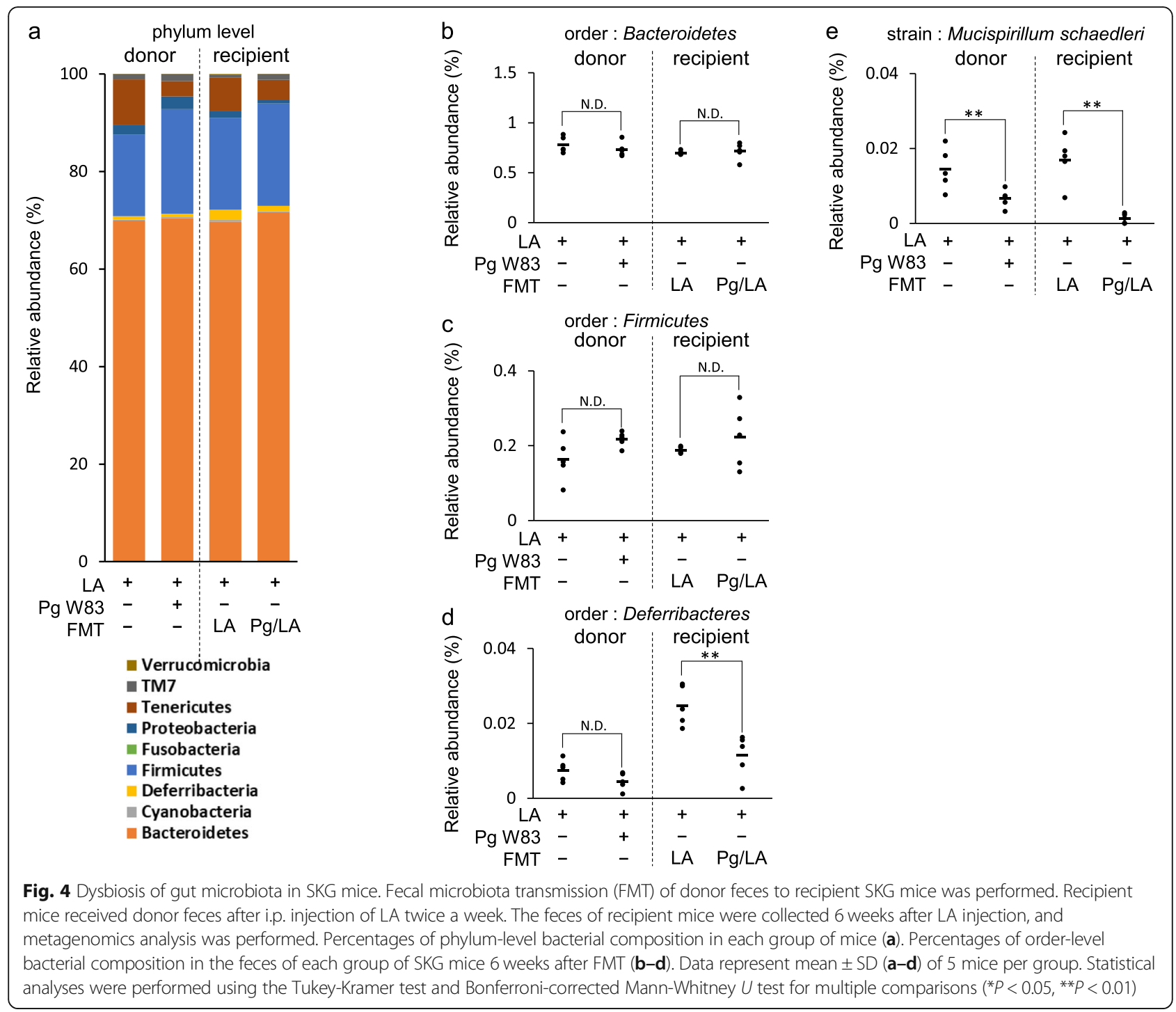

inoculated group in C57BL/6 mice, no differences were observed in the relative abundance of Bacteroides or Firmicutes in donor group as well as recipient groups (Fig. 4b, c) [42]. On the other hand, contrast to Bacteroides and Firmicutes, the relative abundance of Deferribacteres in the gut microbiome of LA-treated recipient group showed significant decrease by FMT from Pg/LA group, compared to that received FMT from LA group (Fig. 4d). While there was no statistically significant difference $(P=0.103)$, LA-treated donor groups showed the similar trend of distribution in relative abundance of Deferribacteres to that found in the FMT recipient mice (Fig. 4d). It is noteworthy that, among Deferribacteres, Mucispirillum schaedleri (also called ASF 457), which represents a commensal bacterium in altered Schaedler flora (ASF) of mice [43, 44], showed the significant decrease in both donor and recipient groups in a Pg-dependent manner (Fig. 4e). These results indicated that oral inoculation of $\mathrm{Pg}$ can affect the gut microbiome, which was represented by the suppression of Deferribacteres abundance, and such trait was also carried over to the FMT recipient mice. As a control of FMT, the buffer used for fecal suspension was inoculated into mice after the LA injection (the control group for the FMT experiment). The microbiome between the LA injected group (donor group) and the control group for the FMT experiment with LA injection did not show the statistical difference (data not shown).

To further assess the effect of FMT on the onset of experimental arthritis, mouse periodontal and joint tissues were analyzed. FMT of donor feces to the recipient mouse was performed through gastric intubation. Therefore, it is conceivable that FMT might have no effect on oral microbiota. To confirm the absence of FMT's effect on oral cavity, alveolar bone healthy mice were morphologically evaluated. FMT did not show any effect on alveolar bone (Figs. S2 and 5a), suggesting that FMT has little or no 


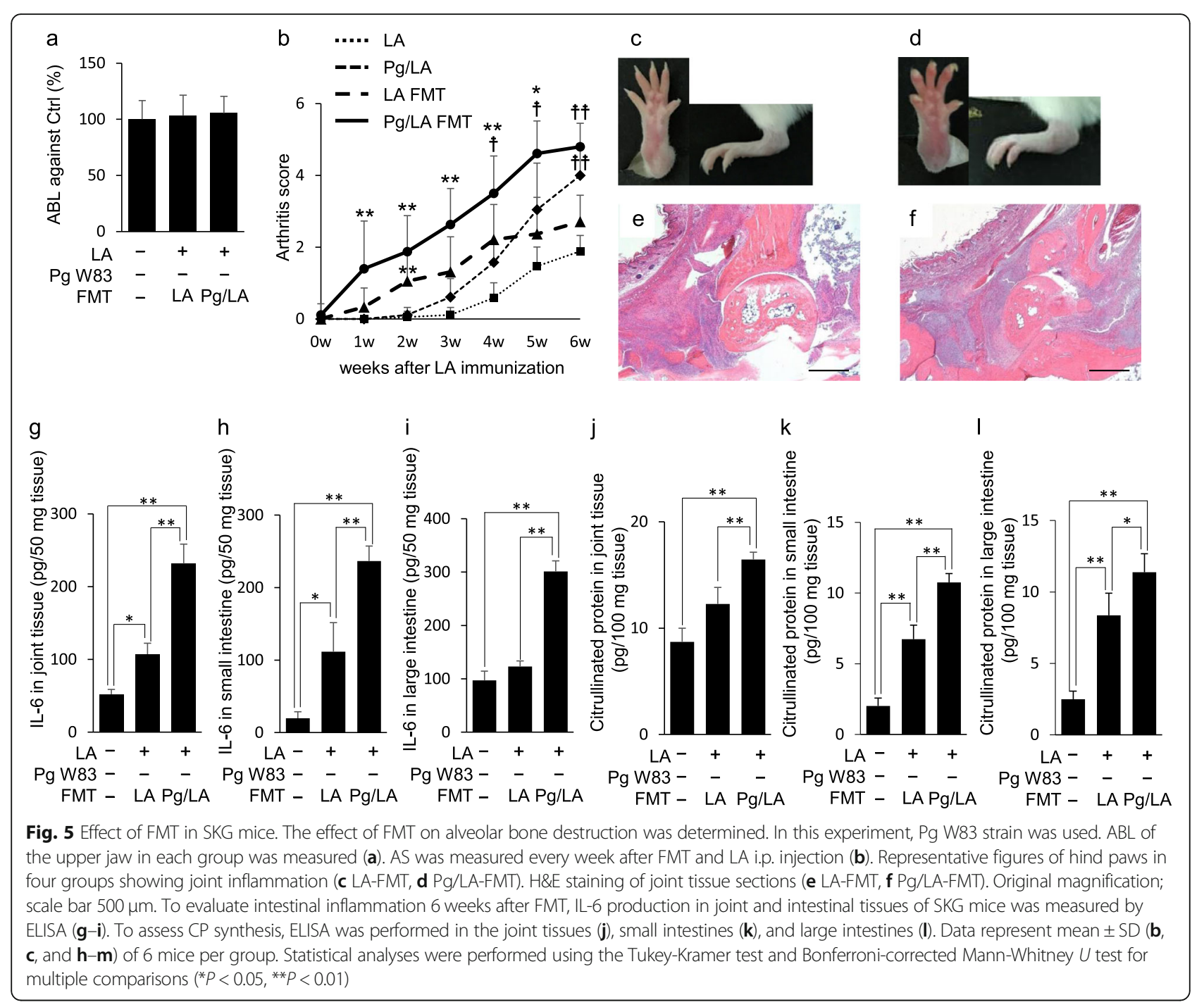

effect on oral microbiome that may cause pathogenic bone resorption. However, severe joint swelling was observed in the LA-FMT and Pg/LA-FMT groups compared to the LA and Pg/LA groups (Fig. 5b-f, Fig. 1c, e). The maximum AS of the LA-FMT and Pg/LA-FMT groups was much higher than that of LA and $\mathrm{Pg} / \mathrm{LA}$, respectively. Pannus formation in the Pg/LA-FMT group was found in joint tissues, similar to that found in the Pg/LA group. Pannus formation and erosion of joint bone tissues were strongly observed in the LA-FMT and Pg/LA-FMT groups (Fig. 5e, f). Additionally, FMT of feces from the LA and Pg/LA groups increased IL-6 in the tissues of joint, small intestine, and large intestine (Fig. $5 \mathrm{~g}-\mathrm{i}$ ). CP levels in the tissues of joint, small intestine, and large intestine were also elevated in the FMT-LA and FMT-Pg/LA groups, with the Pg/LA group showing the highest levels (Fig. 5j-1). The FMT of the feces from the Pg/LA group showed no joint swelling in the absence of LA injection. Similarly, FMT of feces from the Pg group did not show severe arthritis compared with FMT of feces from the LA and $\mathrm{Pg} / \mathrm{LA}$ groups in the presence of LA injection (data not shown).

\section{Effect of PgPAD-deficient mutant on exacerbation of experimental arthritis}

To determine the effect of PgPAD on CP generation and exacerbation of experimental arthritis, the PgPADdeficient Pg 33277 strain as well as Pg 33277 wild type was inoculated into the oral cavity of SKG mice. Bone resorption around the upper molar teeth of Pg 33277 wild-type strain and PgPAD deficient Pg strain was measured (Fig. S3a). Oral inoculation of the PgPADdeficient Pg strain showed slight bone resorption compared to the Ctrl group, which was much lower than that of the wild-type strain (35.2\% lower, Fig. 6a). IL-6 production in gingival tissue of the wild type-inoculated group was elevated. However, this increase was suppressed in the deficient strain (Fig. 6b). Additionally, AS 


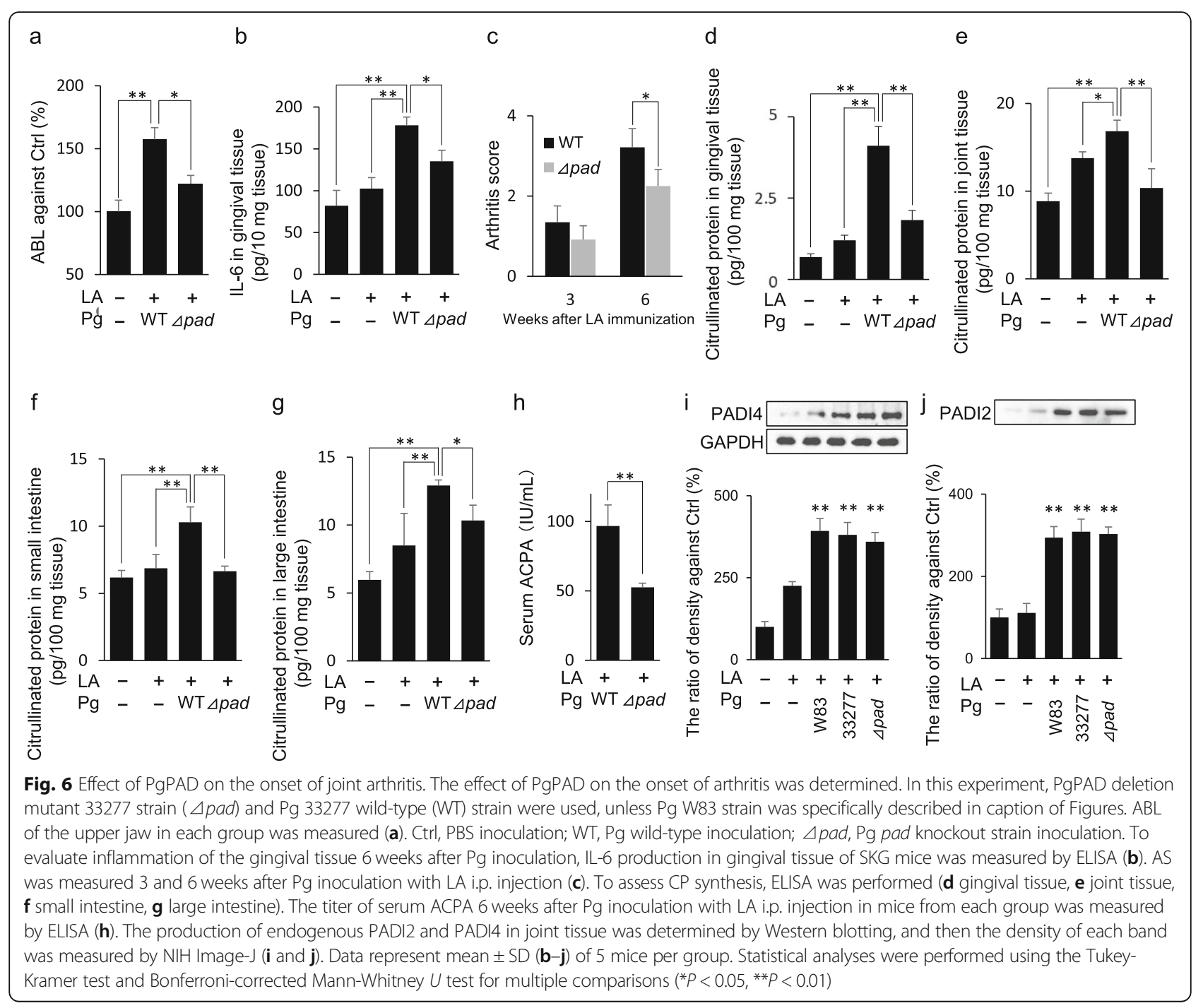

of the PgPAD-deficient Pg strain was significantly lower than the wild-type Pg 33277 strain (Fig. 6c). CP generation in serum and tissues of joint, small intestine, and large intestine by inoculation of the PgPAD-deficient Pg strain was also lower than that of the wild-type strain $(55.6 \%$, $38.2 \%, 35.9 \%$, and $20.2 \%$ decrease, respectively, Fig. $6 \mathrm{~d}-\mathrm{g}$ ). Serum ACPA levels in the PgPAD-deficient Pg strain were significantly decreased compared to Iwild-type (45.8\% decrease, Fig. 6h). The induced production of IL-6 in gingival tissue, $\mathrm{CP}$ generation in gingival tissue, and $\mathrm{CP}$ generation in large intestine by the inoculation of $\mathrm{Pg}$ 33277 wild-type strain was suppressed by the inoculation of PgPAD-deficient Pg 33277 strain. In order to confirm the effect of PgPAD on the progression of arthritis, the internal PAD (PADI2 and PADI4) production in mouse joint tissue was determined. The inoculation of Pg W83, Pg 33277 wild-type, and Pg 33277 PgPAD-deficient strain did not show any difference in the production of PADI2 and PADI4 (Fig. 6i, j). Pg infection in mouse gingival tissue was also confirmed by detection of Pg protein in the gingival tissue homogenates using anti-Pg specific Western blotting (Fig. S3b). In summary, these results using Pg 33277 PgPAD-deficient strain and Pg 33277 wild type demonstrated that Pg PAD is engaged in elevations of citrullinated protein and ACPA without affecting the productions of host's endogenous PADI1 and PADI4, suggesting that PgPAD is responsible for the increased pathogenic outcomes of RA by elevated productions of citrullinated protein and ACPA.

\section{Discussion}

In this study, Pg oral inoculation induced experimental arthritis in SKG mice by altering gut microbiota. FMT from the feces of Pg-inoculated arthritis mice to uninoculated mice caused joint destruction. Concomitantly, CP levels in intestinal and joint tissues were elevated, but partially suppressed in the absence of PgPAD in the PgPAD-deleted mutant group. 
Previous reports showed that periodontitis affected gut microbiota [45]. The inoculation of periodontopathogenic bacteria resulted in the change of gut microbiota and this change induced the destruction of tight junction in the ileum [46]. Human Microbiome Project by genome biology showed that oral cavity and stool bacteria overlapped in nearly $45 \%$ of the subjects [47].

Clinical studies also demonstrated the effect of periodontopathogenic bacteria on gut microbiota [45], likely from the daily inoculation of $1-2 \mathrm{~L}$ of saliva, including pathogenic bacteria (approximately $10^{9} \mathrm{CFU} / \mathrm{mL}$ ) [24]. While planktonic Pg can be eliminated at low $\mathrm{pH}(\mathrm{pH}$ 5), the biofilm of inoculated Pg can survive in acidic conditions as low as artificial gastric juice ( $\mathrm{pH} 3)$ [25].

In this study, Pg inoculation affected gut microbiota composition. Pg inoculation resulted in decreased relative abundance of Deferribacteres and S24-7 in Pg/LA compared to the LA group (Fig. 2d, f). By contrast, the abundance of Bacteroides, Firmicutes, and Clostridiales did not show significant differences among groups, although their relative abundance changed dramatically compared to the control group (Fig. 2). Previous reports showed that Pg administration in the $\mathrm{DBA} / 1 \mathrm{~J}$ experimental arthritis mouse model resulted in decreased relative abundance of Bacteroidetes (phylum level) and Bacteroides (genus level), but increased Firmicutes (phylum level) [25]. In other systemic disease models (type $2 \mathrm{DM}$ in C57BL/6 mice), Pg inoculation increased the abundance of Bacteroides and decreased Firmicutes [46].

However, the administered Pg DNA fragment and protein were not detected in intestinal feces of the Pg and Pg/ LA groups (data not shown). In our previous study, Escherichia coli stimulation did not affect the degree of experimental arthritis, as compared to arthritis without $E$. coli stimulation [15]. Another periodontopathogenic bacterium Prevotella intermedia did not show any differences compared to experimental arthritis without bacterial stimulation [25]. In humans, Prevotella copri was isolated from RA patient samples and identified as a key bacterium [48] involved in arthritis progression. However, in our mouse model, neither $P$. copri nor genetically close bacteria were detected in feces of the arthritis-induced model (data not shown). However, after 6 weeks from oral administration of Pg, the administered Pg DNA fragment was not detected in intestinal tissues and feces of the Pg and $\mathrm{Pg} / \mathrm{LA}$ groups. In the purified DNA from serum, feces, gingival tissue, tongue, lung, stomach, small intestine, and large intestine did address to detect Pg- and $P$. copri-derived DNA fragment by specific primer to $16 \mathrm{~s}$ rRNA. Pg-derived DNA was only detected from gingival tissue, tongue, and lung tissue (Fig. S1d). This is because the bacteria inoculated in this study were the planktonic condition. Therefore, the way of Pg biofilm inoculation should be performed in the future study.
FMT-mediated alteration of gut microbiota composition has been established to treat gastrointestinal disorders, such as Crohn's disease and ulcerative colitis [49]. In FMT-treated patients, an increase in bacterial diversity and abundance of Bacteroides and Firmicutes was observed, along with improved of clinical symptoms. In the mouse model, fecal transplantation from RA patients to mice replicated the human gut microbiota of RA and the onset of experimental arthritis via intestinal Th17 cell activation [22]. In the present study, the FMT from RA-induced donor mice that were orally colonized with $\mathrm{Pg}$, but not control RA-induced donor mice, caused the alteration of gut microbiota in recipient RA-induced mice which, in turn, induced gut inflammation and CP generation, thereby exacerbating arthritis (Figs. S2 and 5). While elevation of $\mathrm{CP}$ generation in the mice received Pg oral inoculation was mediated by PgPAD in a manner independent of host's endogenous PADIs (Fig. 6), FMT that did not contain any Pg could also promote the $\mathrm{CP}$ generation in gut and RA tissue, indicating that dysbiosis of gut microbiota appeared to act on host's endogenous PADIs. Despite limitations of comparing differences between human and mouse microbiota, these studies highlight the importance of gut dysbiosis in the progression of systemic disease, including RA, in the absence of pathogenic oral Pg in gut microbial flora. Both periodontitis and RA treatments resulted in changes in the gut microbiota [45]. Therefore, the monitoring of gut microbiota and specific bacterial species during the treatment of both periodontitis and RA would be useful for identifying risk factors, determining prognosis, and administering therapeutics.

ACPAs target proteins/peptides with citrullinated epitopes and serve as informative RA biomarkers, which are useful for RA diagnosis [50]. ACPAs are generated within synovium and possibly at extra-articular sites prior to disease onset. Recent investigations have begun to elucidate the different mechanisms by which ACPAs may be directly pathogenic in RA. CP is a specific target of ACPA and involved in ACPA generation. This study found increased CP in gingiva, small intestine, large intestine, and joint tissues following Pg inoculation in the experimental arthritis model mouse (Figs. 3, S3, and 6). In humans, four citrullinated autoantigens, including fibrinogen/fibrin, vimentin, $\alpha$-enolase, and type II collagen, are now well accepted as ACPA targets [51-54]. In our study, the specific origin of $\mathrm{CP}$ is unclear. However, $\mathrm{Pg}$ can rapidly generate $\mathrm{CP}$ from $\alpha$-enolase or fibrinogen by proteolytic cleavage at Arg-X peptide bonds using arginine gingipains, followed by citrullination of carboxyterminal arginines by bacterial PAD [13]. Taken together, one of these mechanisms may lead to ACPA generation by PgPAD citrullination. To support this hypothesis, the Pg knockout mutant of PgPAD resulted in 
less $\mathrm{CP}$ generation in serum and in gingival, small and large intestine, and joint tissues (Fig. 6d-g). Furthermore, Pg 33277 wild type-stimulated serum ACPA was diminished by stimulation with the PgPAD knockout mutant (Fig. 6h). However, PADI4, an endogenous PAD, is also involved in CP and ACPA generations, according to the report demonstrating that PADI4 knockout mice decrease severity of experimental autoimmune arthritis [55]. The induction of PADI4 is mediated by TNF- $\alpha$, IL6 , and neutrophil extracellular traps (NETs, NETosis) [55-57]. Increased CP in joint tissue resulted in increased synovial fibroblast migration and spread ability [58]. In lung tissues, CP synthesis and CP-induced inflammation were also reported [59]. Pg inoculation has been shown to induce inflammatory responses in periodontal tissues and in local and systemic sites. In our model, Pg infection activated IL-6 production in the small intestine, large intestine, and joint tissue (Figs. 1i, $\mathrm{j}$, and $3 \mathrm{a}, \mathrm{b})$, resulting in PADI4 induction and subsequent $\mathrm{CP}$ and ACPA generation.

Surprisingly, PgPAD knockout showed both decreased $\mathrm{CP}$ and ACPA production and suppressed inflammation of periodontal tissues, including alveolar bone resorption (Fig. S3a and 6a), which depends on osteoclast activation via RANKL signaling [60]. A previous report showed that citrullinated vimentin induced endogenous PADI4 and RANKL in fibroblast-like synoviocytes derived from RA patients [61]. However, no previous study has shown a correlation between PgPAD and inflammation. Yet, the combination of gingipain and PgPAD generated citrullinated $\alpha$-enolase, and its CP might be an initiator of inflammation. In this present study, Pg 33277 wild type and PgPAD knockout mutant were used. The effects of Pg 33277 on the production of IL-6 and arthritis score showed similar trends to those of Pg W83 (Figs. 1 and 6), suggesting that both stains of Pg can augment the pathogenic outcomes of RA. Importantly, although the production of internal PADI2 and PADI4 level showed no difference among Pg W83, Pg33277, and PgPAD mutant (Fig. 6i, j), the amounts of Pg recovered in gingival tissue among these Pg strains were comparable (Fig. S3b). Therefore, PgPAD has the potential for induction of arthritis. There was a report using PgPAD knockout mutant in the ACPA generation in a mouse model. In that study, alveolar bone resorption, joint swelling, and ACPA production corresponded to our result [62].

\section{Conclusions}

Based on our findings, we propose the following working hypothesis of arthritis pathogenesis with $\mathrm{Pg}$ inoculation (administration) in SKG mice: (1) continued Pg inoculation results in changes in the gut microbiota, (2) dysbiosis of gut microbiota induces inflammation in intestinal tissues, (3) increased $\mathrm{CP}$ in the intestine accelerates systemic ACPA production, (4) severe joint destruction, and (5) CP generation via PgPAD. However, arthritis was also found in the PgPAD knockout mutant, and its AS was higher than that of the normal arthritis model, indicating that Pg is an induction factor for arthritis exacerbation. Therefore, further study is needed to clarify the molecular mechanisms underlying the involvement of Pg infection in the onset of RA.

\section{Supplementary information}

Supplementary information accompanies this paper at https://doi.org/10. 1186/s13075-020-02348-z.

\begin{abstract}
Additional file 1: Figure S1. Assessment of periodontal tissue and evaluation of joint swelling in Pg-administered SKG mice. To determine the effect of Pg infection on periodontal tissue, Pg W83 $\left(10^{8} \mathrm{CFU} / 50 \mathrm{~mL} /\right.$ mouse in $2 \%$ CMC/PBS solution) was administered into the oral cavity of SKG mice (6-8 weeks old, female) twice a week for 42 days. SKG mice also received LA i.p. injection. All mice were sacrificed, and gingival and leg joint tissues were collected. Morphological observation of the mouse upper jaw was analyzed (a). ABL of the upper jaw in each group was measured (b). Joint swelling was quantified using Sakaguchi's AS every week after oral inoculation of Pg $\left(1.0 \times 10^{8} \mathrm{CFU} /\right.$ mouse $)$ and LA i.p. injection (c). Ctrl: PBS inoculation, LA: PBS inoculation + LA i.p. injection (10 $\mu \mathrm{g} /$ mouse), Pg: Pg inoculation, Pg/LA: Pg inoculation + LA i.p. injection. Data represent mean $\pm S D(b, c)$ of 6 mice per group. Data represent mean $\pm S D(b, c)$ of 6 mice per group. Statistical analyses were performed using the Tukey-Kramer test and Bonferroni corrected Mann-Whitney $U$ test for multiple comparisons $(* P<0.05, * * P<0.01)$. After 6 weeks from oral administration of $\mathrm{Pg}, \mathrm{Pg}$-derived DNA fragment by specific primer to $16 \mathrm{~s}$ rRNA in the purified DNA from serum, feces, gingival tissue, tongue, lung, stomach, small intestine, and large intestine (d). Figure S2. Effect of FMT in SKG mice. The effect of FMT on mouse periodontal tissue was determined by monitoring of periodontal bone. Morphological observation of the periodontal alveolar bone of upper jaw of healthy mice was evaluated after 42 days from FMT. Ctrl: PBS inoculation, LA FMT: FMT feces from LA mouse + LA i.p. injection $(10 \mu \mathrm{g} /$ mouse), FMT Pg/LA: FMT feces from $\mathrm{Pg} / \mathrm{LA}$ mouse + LA i.p. injection. Figure S3. Effect of PgPAD on the onset of joint arthritis. The effect of PgPAD on the onset of arthritis was determined (a). Morphological observation of the mouse upper jaw was analyzed. Ctrl: PBS inoculation, Pg WT/LA: Pg wild type inoculation + LA i.p. injection, $\mathrm{Pg} \triangle \mathrm{pad} / \mathrm{LA}$ : Pg pad knockout strain inoculation + LA i.p. injection. The amount of infected Pg was determined by Western blotting (b). Positive control showed $10^{5}$ CFU Pg W83 lysate per well.
\end{abstract}

\section{Abbreviations}

ABL: Alveolar bone loss; AS: Arthritis score; CMC: Carboxymethylcellulose; EDTA: Ethylene diamine tetrameric acid; HE: Hematoxylin and eosin; i.p.: Intraperitoneal; LA: Laminarin; Micro-CT: Micro-computed tomography; PD: Periodontal disease; RA: Rheumatoid arthritis; SEM: Standard error of the mean; SRANKL: Soluble recombinant receptor activation of nuclear factor kappa-B ligand

\section{Acknowledgements}

We would like to thank Editage for English-language editing of this manuscript. We would like to thank Motoyuki Sugai for useful discussions and carefully proofreading the manuscript.

\section{Authors' contributions}

$\mathrm{YH}, \mathrm{KO}, \mathrm{SM}$, and MK established the animal experiments in this study. JH, IK, and $\mathrm{KT}$ analyzed the gut microbiota. MS and KN constructed the knockout mutant of Pg. TO and HK generated the monoclonal antibody against CP. SM, TK, and NM performed the histological experiments. HS and ES evaluated the experimental arthritis symptoms in animal experiments. TF conducted the statistical analysis. HK supervised this study. All authors read and approved the final manuscript. 


\section{Funding}

This research was supported by a Grant-in-Aid for the Encouragement of Young Scientists (B) (19 K18999, 20 K18537), a Grant-in-Aid for Scientific Research (C) (18 K09599) from the Japan Society for the Promotion of Science, and NIH NIDCR grants DE027851 and DE029709.

\section{Availability of data and materials}

The datasets used and/or analyzed during the current study are available from the corresponding author on reasonable request.

\section{Ethics approval and consent to participate}

Animal experiments were approved by the Ethics Committee of Hiroshima University (approval A16-33). All patients provided written informed consent prior to enrollment.

\section{Consent for publication}

The consent of all coauthors was collected before submission.

\section{Competing interests}

The authors declare that they have no competing interests.

\section{Author details}

${ }^{1}$ Department of Periodontal Medicine, Division of Applied Life Sciences, Institute of Biomedical \& Health Sciences, Hiroshima University, 1-2-3 Kasumi, Minami-ku, Hiroshima 734-8553, Japan. ${ }^{2}$ Department of Microbiology and Oral Infection, Graduate School of Biomedical Sciences, Nagasaki University, 1-7-1 Sakamoto, Nagasaki 852-8588, Japan. ${ }^{3}$ Department of Immunology, Faculty of Medicine, Academic Assembly, University of Toyama, 2630 Sugitani, Toyama 930-0194, Japan. ${ }^{4}$ Antimicrobial Resistance Research Center, National Institute of Infectious Diseases (NIID), Toyama 1-23-1, Shinjuku-ku, Tokyo 162-8640, Japan. ${ }^{5}$ Department of Orthodontics and Craniofacial Developmental Biology, Graduate School of Biomedical \& Sciences, Hiroshima University, 1-2-3 Kasumi, Minami-ku, Hiroshima 734-8553, Japan. ${ }^{6}$ Department of Periodontology, Nova Southeastern University College of Dental Medicine, 3200 South University Drive, Fort Lauderdale, FL 33328, USA. ${ }^{7}$ Department of Clinical Immunology and Rheumatology, Hiroshima University Hospital, 1-2-3 Kasumi, Minami-ku, Hiroshima 734-8551, Japan.

\section{Received: 7 January 2020 Accepted: 7 October 2020}

\section{Published online: 19 October 2020}

\section{References}

1. Socransky SS, Haffajee AD. Implications of periodontal microbiology for the treatment of periodontal infections. Compend Suppl. 1994;18:S684-5 688693; quiz S714-687.

2. Hajishengallis G. Immunomicrobial pathogenesis of periodontitis: keystones, pathobionts, and host response. Trends Immunol. 2014;35(1):3-11.

3. Graves D. Cytokines that promote periodontal tissue destruction. $J$ Periodontol. 2008:79(8 Suppl):1585-91.

4. Yoneda M, Naka S, Nakano K, Wada K, Endo H, Mawatari H, Imajo K, Nomura $\mathrm{R}$, Hokamura $\mathrm{K}$, Ono $\mathrm{M}$, et al. Involvement of a periodontal pathogen, Porphyromonas gingivalis on the pathogenesis of non-alcoholic fatty liver disease. BMC Gastroenterol. 2012;12:16.

5. Tervonen T, Oliver RC, Wolff LF, Bereuter J, Anderson L, Aeppli DM. Prevalence of periodontal pathogens with varying metabolic control of diabetes mellitus. J Clin Periodontol. 1994;21(6):375-9.

6. Puertas A, Magan-Fernandez A, Blanc V, Revelles L, O'Valle F, Pozo E, Leon $R$, Mesa F. Association of periodontitis with preterm birth and low birth weight: a comprehensive review. J Matern Fetal Neonatal Med. 2018;31(5):597-602.

7. Laugisch O, Johnen A, Maldonado A, Ehmke B, Burgin W, Olsen I, Potempa J, Sculean A, Duning T, Eick S. Periodontal pathogens and associated intrathecal antibodies in early stages of Alzheimer's disease. J Alzheimers Dis. 2018;66(1):105-14.

8. Paraskevas Kl. Smoking, periodontitis, and Buerger disease. Ann Vasc Surg 2016;33:265

9. Goronzy JJ, Weyand CM. Developments in the scientific understanding of rheumatoid arthritis. Arthritis Res Ther. 2009;11(5):249.

10. Karlson EW, Deane K. Environmental and gene-environment interactions and risk of rheumatoid arthritis. Rheum Dis Clin N Am. 2012;38(2):405-26.
11. Wegner N, Lundberg K, Kinloch A, Fisher B, Malmstrom V, Feldmann M, Venables PJ. Autoimmunity to specific citrullinated proteins gives the first clues to the etiology of rheumatoid arthritis. Immunol Rev. 2010;233(1):34-54.

12. de Pablo P, Chapple IL, Buckley CD, Dietrich T. Periodontitis in systemic rheumatic diseases. Nat Rev Rheumatol. 2009;5(4):218-24.

13. Wegner N, Wait R, Sroka A, Eick S, Nguyen KA, Lundberg K, Kinloch A, Culshaw S, Potempa J, Venables PJ. Peptidylarginine deiminase from Porphyromonas gingivalis citrullinates human fibrinogen and alpha-enolase: implications for autoimmunity in rheumatoid arthritis. Arthritis Rheum. 2010; 62(9):2662-72.

14. Engstrom M, Eriksson K, Lee L, Hermansson M, Johansson A, Nicholas AP, Gerasimcik N, Lundberg K, Klareskog L, Catrina Al, et al. Increased citrullination and expression of peptidylarginine deiminases independently of $\mathrm{P}$. gingivalis and $\mathrm{A}$. actinomycetemcomitans in gingival tissue of patients with periodontitis. J Transl Med. 2018;16(1):214.

15. Yamakawa M, Ouhara K, Kajiya M, Munenaga S, Kittaka M, Yamasaki S, Takeda K, Takeshita K, Mizuno N, Fujita T, et al. Porphyromonas gingivalis infection exacerbates the onset of rheumatoid arthritis in SKG mice. Clin Exp Immunol. 2016;186(2):177-89.

16. Munenaga S, Ouhara K, Hamamoto Y, Kajiya M, Takeda K, Yamasaki S, Kawai T, Mizuno N, Fujita T, Sugiyama E, et al. The involvement of C5a in the progression of experimental arthritis with Porphyromonas gingivalis infection in SKG mice. Arthritis Res Ther. 2018;20(1):247.

17. Clemente JC, Manasson J, Scher JU. The role of the gut microbiome in systemic inflammatory disease. BMJ. 2018;360:j5145.

18. Lin L, Zhang J. Role of intestinal microbiota and metabolites on gut homeostasis and human diseases. BMC Immunol. 2017;18(1):2.

19. De Luca F, Shoenfeld Y. The microbiome in autoimmune diseases. Clin Exp Immunol. 2019;195(1):74-85.

20. Horta-Baas G, Romero-Figueroa MDS, Montiel-Jarquin AJ, Pizano-Zarate ML, Garcia-Mena J, Ramirez-Duran N. Intestinal dysbiosis and rheumatoid arthritis: a link between gut microbiota and the pathogenesis of rheumatoid arthritis. J Immunol Res. 2017;2017:4835189.

21. Kato T, Yamazaki K, Nakajima M, Date Y, Kikuchi J, Hase K, Ohno H, Yamazaki K. Oral administration of porphyromonas gingivalis alters the gut microbiome and serum metabolome. mSphere. 2018;3(5):e00460-18.

22. Maeda Y, Kurakawa T, Umemoto E, Motooka D, Ito Y, Gotoh K, Hirota K, Matsushita M, Furuta Y, Narazaki M, et al. Dysbiosis contributes to arthritis development via activation of autoreactive T cells in the intestine. Arthritis Rheumatol. 2016;68(11):2646-61.

23. Lorenzo D, GianVincenzo Z, Carlo Luca R, Karan G, Jorge V, Roberto M, Javad P. Oral-gut microbiota and arthritis: is there an evidence-based axis? 」 Clin Med. 2019;8(10).

24. Pietiainen M, Liljestrand JM, Kopra E, Pussinen PJ. Mediators between oral dysbiosis and cardiovascular diseases. Eur J Oral Sci. 2018;126(Suppl 1):26-36.

25. Sato K, Takahashi N, Kato T, Matsuda Y, Yokoji M, Yamada M, Nakajima T, Kondo N, Endo N, Yamamoto R, et al. Aggravation of collagen-induced arthritis by orally administered Porphyromonas gingivalis through modulation of the gut microbiota and gut immune system. Sci Rep. 2017;7(1):6955.

26. van Venrooij WJ, van Beers JJ, Pruijn GJ. Anti-CCP antibodies: the past, the present and the future. Nat Rev Rheumatol. 2011;7(7):391-8.

27. Sakaguchi N, Takahashi T, Hata H, Nomura T, Tagami T, Yamazaki S, Sakihama T, Matsutani T, Negishi I, Nakatsuru S, et al. Altered thymic T-cell selection due to a mutation of the ZAP-70 gene causes autoimmune arthritis in mice. Nature. 2003:426(6965):454-60.

28. Naito M, Tominaga T, Shoji M, Nakayama K. PGN_0297 is an essential component of the type IX secretion system (T9SS) in Porphyromonas gingivalis: Tn-seq analysis for exhaustive identification of T9SS-related genes. Microbiol Immunol. 2019;63(1):11-20.

29. Shoji M, Sato K, Yukitake H, Kamaguchi A, Sasaki Y, Naito M, Nakayama K. Identification of genes encoding glycosyltransferases involved in lipopolysaccharide synthesis in Porphyromonas gingivalis. Mol Oral Microbiol. 2018;33(1):68-80.

30. Kawai T, Paster BJ, Komatsuzawa H, Ernst CW, Goncalves RB, Sasaki H, Ouhara K, Stashenko PP, Sugai M, Taubman MA. Cross-reactive adaptive immune response to oral commensal bacteria results in an induction of receptor activator of nuclear factor-kappaB ligand (RANKL)-dependent periodontal bone resorption in a mouse model. Oral Microbiol Immunol. 2007;22(3):208-15

31. Hata H, Sakaguchi $N$, Yoshitomi H, Iwakura Y, Sekikawa K, Azuma Y, Kanai C, Moriizumi E, Nomura T, Nakamura T, et al. Distinct contribution of IL-6, TNF- 
alpha, IL-1, and IL-10 to T cell-mediated spontaneous autoimmune arthritis in mice. J Clin Invest. 2004;114(4):582-8.

32. Tsuda R, Ozawa T, Kobayashi E, Hamana H, Taki H, Tobe K, Sugiyama E, Iwamoto M, Imura J, Kishi H, et al. Monoclonal antibody against citrullinated peptides obtained from rheumatoid arthritis patients reacts with numerous citrullinated microbial and food proteins. Arthritis Rheumatol. 2015;67(8):2020-31.

33. Mukai T, Gallant R, Ishida S, Kittaka M, Yoshitaka T, Fox DA, Morita Y, Nishida K, Rottapel R, Ueki Y. Loss of SH3 domain-binding protein 2 function suppresses bone destruction in tumor necrosis factor-driven and collageninduced arthritis in mice. Arthritis Rheumatol. 2015;67(3):656-67.

34. Matsuki T, Watanabe K, Fujimoto J, Miyamoto Y, Takada T, Matsumoto K, Oyaizu H, Tanaka R. Development of $16 \mathrm{~S}$ rRNA-gene-targeted group-specific primers for the detection and identification of predominant bacteria in human feces. Appl Environ Microbiol. 2002;68(11):5445-51.

35. Ouhara K, Iwasaki Y, Kajiya M, Savitri IJ, Kitagawa M, Tokunaga N, Shintani T, Ogawa I, Hino T, Fujita T, et al. The differential expression of mgl mRNA by Porphyromonas gingivalis affects the production of methyl mercaptan. Oral Dis. 2015;21(5):626-33.

36. Andersson AF, Lindberg M, Jakobsson H, Backhed F, Nyren P, Engstrand L. Comparative analysis of human gut microbiota by barcoded pyrosequencing. PLoS One. 2008;3(7):e2836.

37. Caporaso JG, Kuczynski J, Stombaugh J, Bittinger K, Bushman FD, Costello EK, Fierer N, Pena AG, Goodrich JK, Gordon Jl, et al. QIIME allows analysis of highthroughput community sequencing data. Nat Methods. 2010;7(5):335-6.

38. Edgar RC. Search and clustering orders of magnitude faster than BLAST. Bioinformatics. 2010;26(19):2460-1.

39. Ortiz P, Bissada NF, Palomo L, Han YW, Al-Zahrani MS, Panneerselvam A, Askari A. Periodontal therapy reduces the severity of active rheumatoid arthritis in patients treated with or without tumor necrosis factor inhibitors. J Periodontol. 2009;80(4):535-40.

40. Yoshitomi H, Sakaguchi N, Kobayashi K, Brown GD, Tagami T, Sakihama T, Hirota K, Tanaka S, Nomura T, Miki I, et al. A role for fungal \{beta\}-glucans and their receptor Dectin-1 in the induction of autoimmune arthritis in genetically susceptible mice. J Exp Med. 2005;201(6):949-60.

41. Shiomi A, Usui T, Ishikawa Y, Shimizu M, Murakami K, Mimori T. GM-CSF but not IL-17 is critical for the development of severe interstitial lung disease in SKG mice. J Immunol. 2014;193(2):849-59.

42. Nakajima M, Arimatsu K, Kato T, Matsuda Y, Minagawa T, Takahashi N, Ohno $H$, Yamazaki K. Oral administration of $P$. gingivalis induces dysbiosis of gut microbiota and impaired barrier function leading to dissemination of Enterobacteria to the liver. PLoS One. 2015;10(7):e0134234.

43. Dewhirst FE, Chien CC, Paster BJ, Ericson RL, Orcutt RP, Schauer DB, Fox JG. Phylogeny of the defined murine microbiota: altered Schaedler flora. Appl Environ Microbiol. 1999;65(8):3287-92.

44. Wymore Brand M, Wannemuehler MJ, Phillips GJ, Proctor A, Overstreet AM, Jergens AE, Orcutt RP, Fox JG. The altered Schaedler Flora: continued applications of a defined murine microbial community. ILAR J. 2015;56(2):169-78.

45. Olsen I, Yamazaki K. Can oral bacteria affect the microbiome of the gut? J Oral Microbiol. 2019;11(1):1586422

46. Arimatsu K, Yamada H, Miyazawa H, Minagawa T, Nakajima M, Ryder MI, Gotoh K, Motooka D, Nakamura S, lida T, et al. Oral pathobiont induces systemic inflammation and metabolic changes associated with alteration of gut microbiota. Sci Rep. 2014;4:4828.

47. Segata N, Haake SK, Mannon P, Lemon KP, Waldron L, Gevers D, Huttenhower C, Izard J. Composition of the adult digestive tract bacterial microbiome based on seven mouth surfaces, tonsils, throat and stool samples. Genome Biol. 2012;13(6):R42.

48. Pianta A, Arvikar S, Strle K, Drouin EE, Wang Q, Costello CE, Steere AC. Evidence of the immune relevance of Prevotella copri, a gut microbe, in patients with rheumatoid arthritis. Arthritis Rheumatol. 2017;69(5): 964-75.

49. Kellermayer R. Fecal microbiota transplantation: great potential with many challenges. Transl Gastroenterol Hepatol. 2019;4:40.

50. Aggarwal R, Liao K, Nair R, Ringold S, Costenbader KH. Anti-citrullinated peptide antibody assays and their role in the diagnosis of rheumatoid arthritis. Arthritis Rheum. 2009;61(11):1472-83.

51. Vossenaar ER, Despres N, Lapointe E, van der Heijden A, Lora M, Senshu T, van Venrooij WJ, Menard HA. Rheumatoid arthritis specific anti-Sa antibodies target citrullinated vimentin. Arthritis Res Ther. 2004;6(2):R142-50.
52. Kinloch A, Tatzer V, Wait R, Peston D, Lundberg K, Donatien P, Moyes D, Taylor PC, Venables PJ. Identification of citrullinated alpha-enolase as a candidate autoantigen in rheumatoid arthritis. Arthritis Res Ther. 2005;7(6): R1421-9.

53. Masson-Bessiere C, Sebbag M, Girbal-Neuhauser E, Nogueira L, Vincent C, Senshu T, Serre G. The major synovial targets of the rheumatoid arthritisspecific antifilaggrin autoantibodies are deiminated forms of the alpha- and beta-chains of fibrin. J Immunol. 2001;166(6):4177-84.

54. Burkhardt H, Sehnert B, Bockermann R, Engstrom A, Kalden JR, Holmdahl R. Humoral immune response to citrullinated collagen type II determinants in early rheumatoid arthritis. Eur J Immunol. 2005;35(5):1643-52.

55. Suzuki A, Kochi Y, Shoda H, Seri Y, Fujio K, Sawada T, Yamada R, Yamamoto $K$. Decreased severity of experimental autoimmune arthritis in peptidylarginine deiminase type 4 knockout mice. BMC Musculoskelet Disord. 2016;17:205

56. Yang Y, Dai M. Expression of PADI4 in patients with ankylosing spondylitis and its role in mediating the effects of TNF-alpha on the proliferation and osteogenic differentiation of human mesenchymal stem cells. Int J Mol Med. 2015;36(2):565-70.

57. Yahagi A, Saika T, Hirano H, Takai-Imamura M, Tsuji F, Aono H, Iseki M, Morita Y, Igarashi H, Saeki Y, et al. IL-6-PAD4 axis in the earliest phase of arthritis in knock-in gp130F759 mice, a model for rheumatoid arthritis. RMD Open. 2019;5(2):e000853.

58. Sipila KH, Ranga V, Rappu P, Mali M, Pirila L, Heino I, Jokinen J, Kapyla J, Johnson MS, Heino J. Joint inflammation related citrullination of functional arginines in extracellular proteins. Sci Rep. 2017;7(1):8246.

59. Catrina Al, Ytterberg AJ, Reynisdottir G, Malmstrom V, Klareskog L. Lungs, joints and immunity against citrullinated proteins in rheumatoid arthritis. Nat Rev Rheumatol. 2014;10(11):645-53.

60. Taubman MA, Valverde P, Han X, Kawai T. Immune response: the key to bone resorption in periodontal disease. J Periodontol. 2005;76(11 Suppl): 2033-41.

61. Miao CG, Yang YY, He X, Li XF, Huang C, Huang Y, Zhang L, Lv XW, Jin Y, Li J. Wnt signaling pathway in rheumatoid arthritis, with special emphasis on the different roles in synovial inflammation and bone remodeling. Cell Signal. 2013;25(10):2069-78.

62. Gully N, Bright R, Marino V, Marchant C, Cantley M, Haynes D, Butler C, Dashper S, Reynolds E, Bartold M. Porphyromonas gingivalis peptidylarginine deiminase, a key contributor in the pathogenesis of experimental periodontal disease and experimental arthritis. PLoS One. 2014;9(6):e100838

\section{Publisher's Note}

Springer Nature remains neutral with regard to jurisdictional claims in published maps and institutional affiliations.

Ready to submit your research? Choose BMC and benefit from:

- fast, convenient online submission

- thorough peer review by experienced researchers in your field

- rapid publication on acceptance

- support for research data, including large and complex data types

- gold Open Access which fosters wider collaboration and increased citations

- maximum visibility for your research: over $100 \mathrm{M}$ website views per year

At BMC, research is always in progress.

Learn more biomedcentral.com/submissions 